\title{
Comparison of long-term low-fat versus high-fat diets on blood lipids: a systematic review and meta-analysis
}

\author{
L. Schwingshackl and G. Hoffmann \\ University of Vienna, Faculty of Life Sciences, Department of Nutritional Sciences, A-1090 Vienna, Austria
}

Dietary fat plays an important role in the primary prevention of cardiovascular disease, but long-term effects of different percentages of fat in the diet on blood lipids as biomarkers of cardiovascular risk remain to be established. In the present systematic review, randomized controlled intervention trials (RCTs) investigating the long-term effects of low fat (LF) diets as compared to diets with high amounts of fat (HF) on blood lipids were analyzed. Three electronic databases (MEDLINE, EMBASE, and the Cochrane Trial Register) were searched for RCTs with no restriction to calendar date or language. Moreover, reference lists from retrieved articles as well as systematic reviews and meta-analysis were reviewed. RCTs reporting mean post or mean differences with their standard deviation for effects of LF vs. HF diets on blood lipids were included according to the Cochrane Handbook ${ }^{(1)}$. Both authors independently extracted data and assessed study quality with the Jadad Score. Study specific weighted mean differences were pooled using a random effect model (REVIEW MANAGER 5.1.0). For RCTs with a quality score $\geq 3$, an additional sensitivity analysis was performed. By using the metareg function of STATA 12.0 a random effect meta-regression was calculated to investigate high heterogeneity.
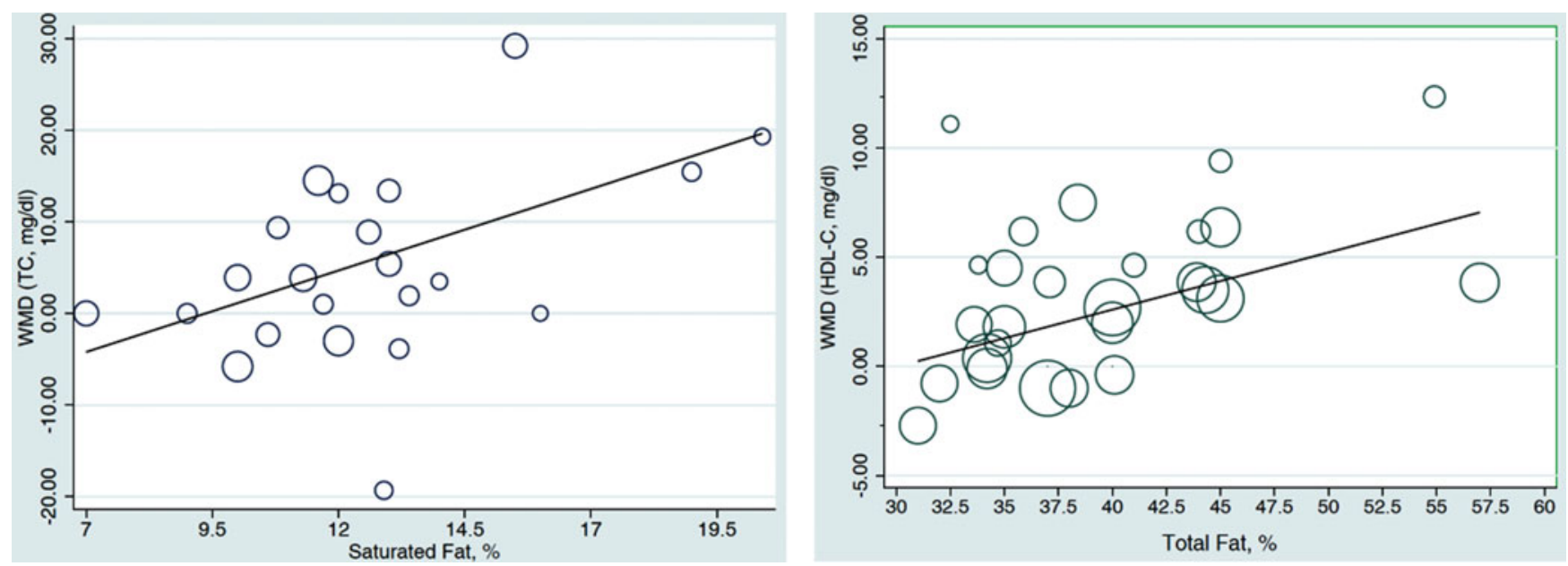

Fig. 1. Bubble plots for the association between saturated fatty acids and total cholesterol (left panel), and between total fat HDL-cholesterol (right panel).

A total of 32 studies were included in the primary meta-analysis. Decrease in total cholesterol (TC) $[-4.55 \mathrm{mg} / \mathrm{dl}(95 \% \mathrm{CI}-8.03$ to $-1.07)(p=0.01)$ ] and LDL-cholesterol [weighted mean differences (WMD): $-3.11 \mathrm{mg} / \mathrm{dl}(95 \% \mathrm{CI}-4.51$ to -1.71$), p<0.0001$ ] was significantly more pronounced following LF diets, while raise in HDL-cholesterol [WMD: $2.35 \mathrm{mg} / \mathrm{dl}(95 \%$ CI 1.92 to 3.42$), p<0.0001$ ] and reduction in triacylglycerols (TG) [WMD: $-8.38 \mathrm{mg} / \mathrm{dl}(95 \% \mathrm{CI}-13.50$ to -3.25$), p=0.001$ ] was more distinct in the HF groups. Sensitivity analysis could confirm the results of the primary analyses. In addition, exclusion of the largest study ${ }^{(2)}$ to account for a potential "large study effect" on the pooled WMD did not yield an outcome significantly different from the comprehensive meta-analyses. Post-hoc meta-regression indicated that changes in TC were associated with higher amounts of saturated fat and lower contents in polyunsaturated fat, whereas changes in HDL-cholesterol were related to higher amounts of total fat and monounsaturated fat in HF diets, respectively. Thus, neither LF nor HF showed definite beneficial effects on blood lipids which is why dietary recommendations in either direction should be considered carefully.

1. Higgins JP \& Green S (2011) Cochrane Handbook of systematic reviews, Version 5.1.0 http://www.cochrane-handbook.org (accessed June 2012).

2. Howard BV, Curb JD, Eaton CB, et al. (2010) Low-fat dietary pattern and lipoprotein risk factors: the Women's Health Initiative Dietary Modification Trial. Am J Clin Nutr 9, 860-874. 